\title{
Оптическая спектроскопия резонансной брэгговской структуры с квантовыми ямами InGaN/GaN
}

\author{
(C) А.С. Большаков, В.В. Чалдышев, Е.Е. Заварин, А.В. Сахаров, В.В. Лундин, А.Ф. Цацульников \\ Физико-технический институт им. А.Ф. Иофрфе Российской академии наук, \\ 194021 Санкт-Петербург, Россия \\ E-mail: chald.gvg@mail.ioffe.ru
}

(Получена 27 апреля 2016 г. Принята к печати 10 мая 2016 г.)

Исследованы спектры оптического отражения и пропускания при комнатной температуре периодической полупроводниковой гетероструктуры с 60 квантовыми ямами $\mathrm{InGaN} / \mathrm{GaN}$. Период структуры подбирался так, чтобы при определенных углах падения света обеспечить совпадение энергии фотона, испытывающего резонансное брэгговское отражение, с энергией возбуждения экситонов в квантовых ямах. Подгонка спектров, измеренных при углах падения света 30 и $60^{\circ}$, с учетом как резонансных экситонных переходов, так и переходов в непрерывный спектр состояний квантовой ямы позволила определить параметры экситонов в квантовых ямах. Получено значение параметра радиационного затухания $(0.20 \pm 0.02)$ мэВ.

\section{1. Введение}

Резонансные брэгговские структуры с квантовыми ямами (КЯ) представляют собой периодические полупроводниковые гетероструктуры с периодом, при котором длина волны брэгговского резонанса в отражении соответствует энергии возбуждения экситона. Такие системы примечательны тем, что в них должно наблюдаться уменьшение излучательного времени жизни экситонов в КЯ пропорционально числу периодов $[1,2]$, выражающееся в усилении экситонного вклада в отражение. Это усиление экспериментально наблюдалось в системах КЯ CdTe/CdMgTe [3], CdTe/CdZnTe [4], InGaAs/ GaAs [5,6], GaAs/AlGaAs [7-10], InGaN/GaN [11,12]. Большая энергия связи экситонов в $\mathrm{GaN}(\sim 25$ мэВ в объемном материале по сравнению с $\sim 4$ мэВ в объемном GaAs), увеличенная за счет размерного квантования, в сочетании с усилением экситонного отражения в брэгговской структуре позволили отчетливо наблюдать экситонный вклад в отражение при комнатной температуре [11]. Параметр радиационного затухания экситона в КЯ, определяющий силу оптического отклика экситона, был оценен в [11] в 0.17 мэВ, что также значительно больше, чем в КЯ на основе GaAs, $\sim(0.03 \pm 0.04)$ мэВ. Эта оценка была выполнена на основании подгонки спектра отражения, полученного в условиях двойного резонанса. Однако общие соображения подсказывают, что подгонка брэгговской особенности по одному лишь спектру может быть неоднозначной, в частности может быть неверно оценено соотношение между пассивным диэлектрическим контрастом (со слабой дисперсией) и сильно зависящим от длины волны экситонным вкладом в диэлектрический контраст.

В данной работе проведено дальнейшее исследование образца, содержащего периодическую систему 60 Кя InGaN/GaN [11], с целью наилучшего определения таких параметров экситонов в КЯ, как радиационное и нерадиационное затухание. Для выполнения этих задач измерения и численное моделирование проводились при разных углах падения света. Поскольку положение брэгговского максимума зависит от длины волны света, а положение экситонных резонансов не зависит, если не учитывать слабого эффекта пространственной дисперсии, таким путем можно исследовать модификацию оптических спектров в зависимости от отстройки брэгговского резонанса от частоты возбуждения экситонов в КЯ.

\section{2. Исследованный образец и методика эксперимента}

Образец представлял собой набор из 60 эквидистантных КЯ InGaN/GaN, выращенный на подложке из сапфира (рис. 1). Для избежания прорастания дислокаций в рабочую область образца между ней и подложкой был выращен буферный слой $\mathrm{GaN}$ толщиной $\sim 2.5$ мкм. Толщина КЯ $\mathrm{InGaN}$ составляла $\sim 2$ нм, период структуры был $\sim 80$ нм. Ростовой процесс проводился без вращения подложкодержателя, поэтому эпитаксиальные слои имели некоторую неоднородность по толщине и составу, вследствие чего оптические свойства разных

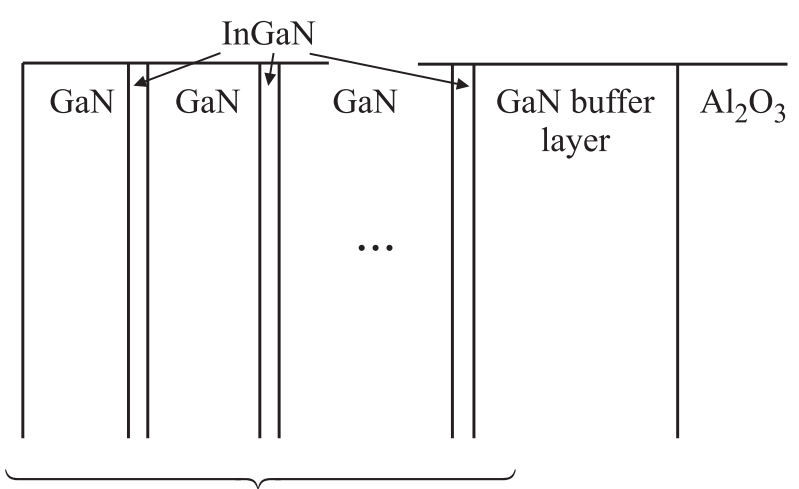

60 periods

Рис. 1. Эскиз исследованной структуры. 
областей поверхности образца различались. Детали процесса изготовления образца описаны в [13].

Измерение спектров отражения и пропускания проводилось по стандартной схеме с использованием дейтериевой лампы Hamamatsu L2D2 (типа L6565) в качестве источника света, спектрометра OceanOptics HR4000CG-UV-NIR, стыкуемого с ним оптического волокна OceanOptics типа „solarization resistant“, а также фокусирующих линз из кварцевого стекла. В качестве поляризатора применялась призма Глана-Тейлора либо тонкопленочный поляризатор. Нормировка измеренных спектров отражения проводилась при помощи опорного спектра отражения от сапфира с учетом коэффициентов Френеля. Нормировку спектров пропускания напрямую осуществить не удавалось вследствие того, что задняя поверхность образца была шероховатой и значительно рассеивала свет. Нормировка отдельных спектров пропускания проводилась на основе подгонки одновременно с соответствующим спектром отражения.

\section{3. Детали расчета}

Компьютерное моделирование (подгонка) спектров выполнялась с использованием метода матриц переноса. Матрицы переноса через слои КЯ строились на основе формул экситонного отражения и пропускания, приведенных в работе [14]. При этом вследствие малости эффекта не учитывалось изменение резонансной частоты экситона с изменением угла падения света. Зависимость параметра радиационного затухания $\Gamma_{0}$ от угла падения света на образец $\varphi$ бралась в виде

$$
\Gamma_{0}(\varphi)=\Gamma_{0}\left(0^{\circ}\right) \operatorname{Re}\left[\frac{n_{\mathrm{QW}}}{\sqrt{n_{\mathrm{QW}}^{2}-\sin ^{2} \varphi}}\right]
$$

для $s$-поляризации и

$$
\Gamma_{0}(\varphi)=\Gamma_{0}\left(0^{\circ}\right) \operatorname{Re}\left[\frac{\sqrt{n_{\mathrm{QW}}^{2}-\sin ^{2} \varphi}}{n_{\mathrm{QW}}}\right]
$$

для $p$-поляризации, где $\Gamma_{0}\left(0^{\circ}\right)-$ радиационное затухание экситона для нормального падения света, $n_{\mathrm{QW}}=\sqrt{\varepsilon(\omega)}-$ фоновый показатель преломления в КЯ.

Помимо экситонного вклада, учитывалось поглощение в непрерывный спектр состояний КЯ. Для этого в фоновую (без учета экситонного вклада) диэлектрическую функцию $\varepsilon(\omega)$ КЯ вводился дополнительный член [15]:

$$
\begin{aligned}
& \varepsilon(\omega)=\varepsilon\left(\omega^{\prime}\right)+N f_{b} \\
& \times\left[\frac{\arctan \left(\omega_{b} / \sqrt{i \gamma \omega^{\prime}-\omega^{\prime 2}}\right)}{\sqrt{i \gamma \omega^{\prime}-\omega^{\prime 2}}}-\frac{\arctan \left(\omega_{b} / \sqrt{i \gamma \omega-\omega^{2}}\right)}{\sqrt{i \gamma \omega-\omega^{2}}}\right],
\end{aligned}
$$

где $\omega-$ частота света, $\varepsilon\left(\omega^{\prime}\right)-$ диэлектрическая проницаемость на некоторой частоте $\omega^{\prime}$, являющаяся подгоночным параметром задачи, $N$ - комбинированная плотность состояний для оптических переходов между зонами размерного квантования дырок и электронов, $f_{b}$ - коэффициент, пропорциональный силе осциллятора указанных переходов, $\gamma$ - параметр неоднородного уширения для указанных переходов, $\hbar \omega_{b}-$ энергия края оптических переходов между зонами размерного квантования дырок и электронов без учета экситонного эффекта.

Если ввести коэффициент $f_{\text {ех }}$, связанный с эффективным продольно-поперечным расщеплением экситона $\omega_{\mathrm{eff}}$ посредством соотношения $f_{\text {ex }}=2 \varepsilon\left(\omega^{\prime}\right) \omega_{0} \omega_{\text {eff, }}$, то отношение $f_{\text {ex }} / N f_{b}$ должно составлять 2 и 16 экситонных ридбергов, деленных на $\hbar$, для трехмерной и идеальной двумерной систем соответственно [15]. Эффективное продольно-поперечное расщепление экситона выражается формулой [16]

$$
\omega_{\mathrm{eff}}=\frac{2 \Gamma_{0}}{\sin \left[q\left(\omega_{0}\right) a\right]},
$$

где $q\left(\omega_{0}\right)$ - волновой вектор света на частоте экситонного резонанса, $a-$ ширина КЯ.

Возможность рэлеевского рассеяния на дислокациях и малоугловых границах в буферном слое учитывалась добавкой в мнимую часть показателя преломления этого слоя слагаемого, обратно пропорционального кубу длины волны света. При этом соответствующий вклад в коэффициент поглощения оказывается обратно пропорциональным четвертой степени длины волны, в соответствии с законом Рэлея.

\section{4. Результаты и обсуждение}

На рис. $2, a, b$ показаны экспериментально измеренные (кривые 1) и расчетные (кривые 2,3) спектры отражения при углах падения $s$-поляризованного света 30 и $60^{\circ}$. Bсе результаты получены при комнатной температуре. Представлены результаты расчета с учетом как экситонного резонанса в КЯ, так и поглощения в непрерывном спектре электронных и дырочных состояний в КЯ (кривае 2), а также с учетом только экситонного резонанса (кривая 3). В спектрах наблюдаются осцилляции Фабри-Перо, происходящие вследствие интерференции света, отраженного от границ всей эпитаксиальной структуры. Осцилляции затухают в коротковолновой области вследствие поглощения в КЯ и барьерах $\mathrm{GaN}$. На фоне осцилляций Фабри-Перо выделяется пик отражения при выполнении брэгговского условия. С увеличением угла падения света пик, в согласии с законом Вульфа-Брэгга, смещается в коротковолновую область. Соответствующие спектры пропускания показаны на рис. $3, a, b$. На них также видны осцилляции Фабри-Перо, затухающие в коротковолновой области. Вследствие поглощения в эпитаксиальной структуре одновременно с затуханием осцилляций усиливается спад пропускания. На длине волны брэгговского резонанса 

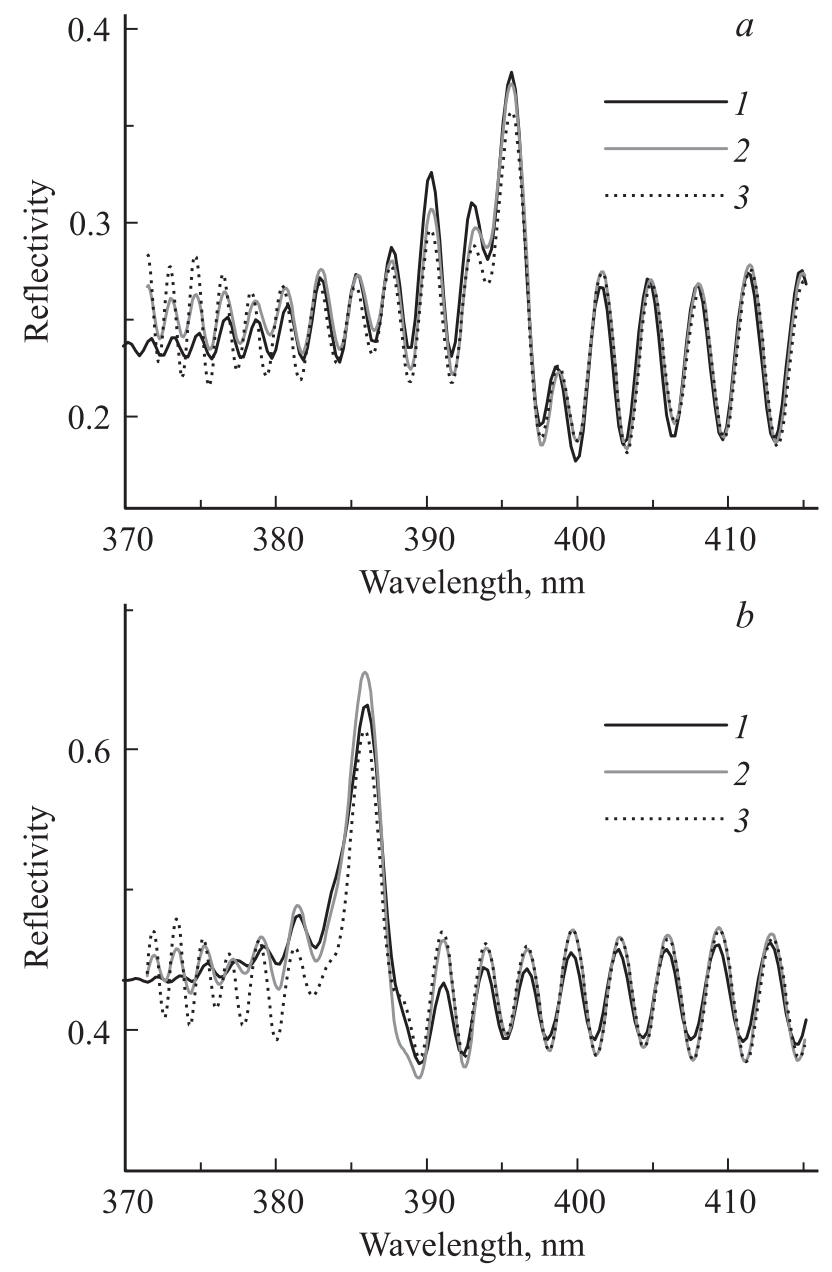

Рис. 2. Спектры отражения исследованной структуры. 1 - экспериментальный спектр, 2 - расчетный спектр с учетом поглощения в непрерывном спектре КЯ, 3 - расчетный спектр с учетом только экситонного резонанса. Угол падения света $30(a)$ и $60^{\circ}(b), s$-поляризация, комнатная температура.

также наблюдается особенность, но существенно более слабая, чем в спектре отражения.

Представленные на рис. 2 и 3 результаты расчета соответствуют одним и тем же параметрам модели. Как можно видеть, учет поглощения на состояниях непрерывного спектра КЯ позволяет лучше описать экспериментальные данные в коротковолновой области. Остающееся расхождение можно объяснить наличием в КЯ дополнительных подзон и соответствующих им экситонных состояний, а также хвостом плотности состояний в барьерах $\mathrm{GaN}$, что не учитывалось в модели. Нужно отметить, что учет одного лишь экситонного резонанса привел бы к некоторой переоценке радиационного затухания экситона (полученное в [11] меньшее значение объясняется тем, что подгонялся только один спектр).

В длинноволновой области спектров отражения (на рисунках не показана) наблюдался монотонный рост амплитуды осцилляций Фабри-Перо. Поскольку в этой области не должно наблюдаться существенного поглощения, этот факт был учтен в модели посредством введения рэлеевского рассеяния в буферном слое $\mathrm{GaN}$. Нужно отметить, что вследствие сильного рассеяния на задней шероховатой поверхности сапфировой подложки спектры пропускания не могли быть нормированы напрямую. Поэтому после нормировки на спектр источника света спектры пропускания умножались на некоторые линейные функции для учета рассеяния на задней поверхности образца и возможных хроматических аберраций в оптической системе. Коэффициенты линейных функций подбирались до наилучшего совпадения с расчетными спектрами.

Основные использованные в расчете параметры следующие: радиационное затухание экситона 0.20 мэВ, нерадиационное затухание экситона 40 мэВ, энергия возбуждения экситона 3.24 эВ (на $\sim 10$ мэВ больше, чем следует из данных по фотолюминесценции данной обла-
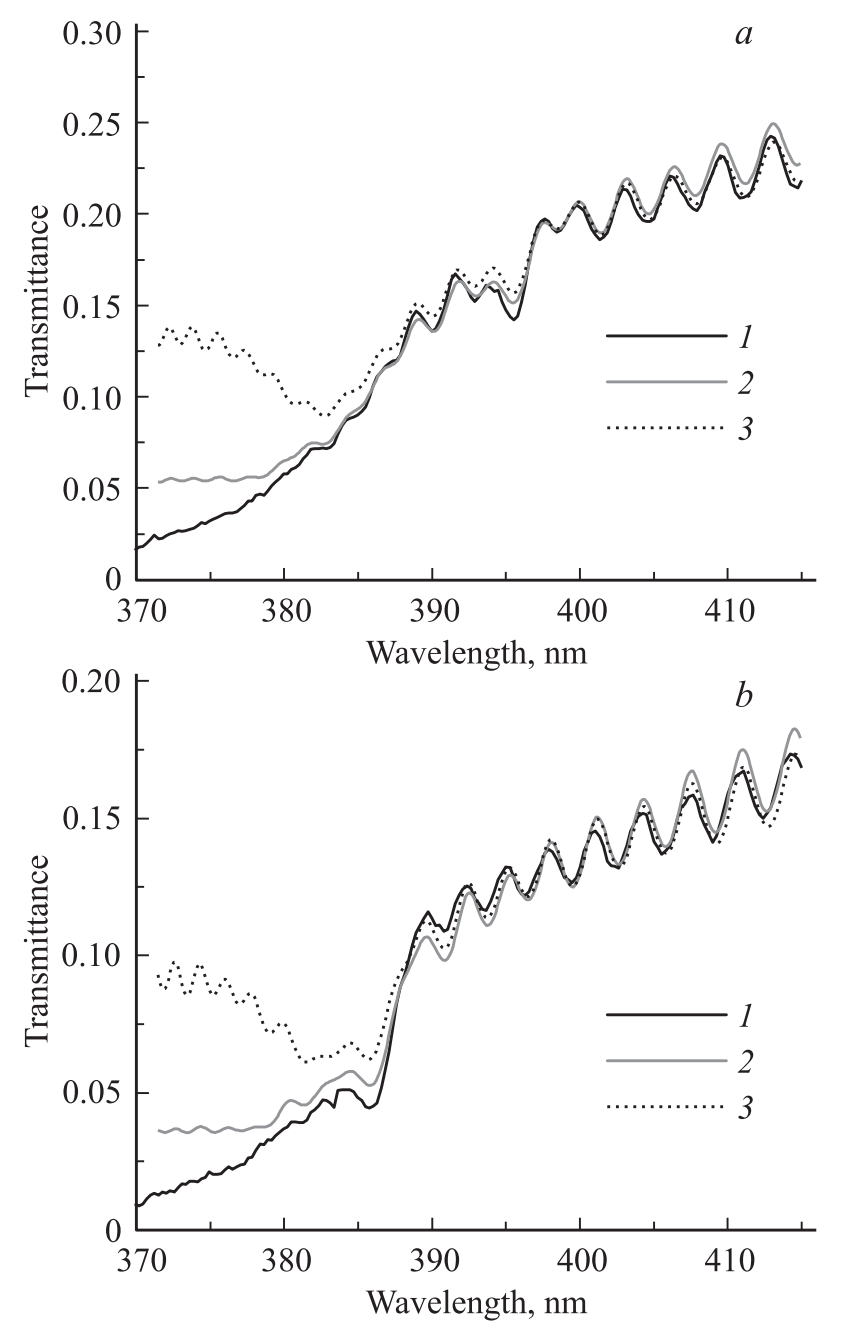

Рис. 3. Спектры пропускания исследованной структуры. 1 экспериментальный спектр, 2 - расчетный спектр с учетом поглощения в непрерывном спектре КЯ, 3 - расчетный спектр с учетом только экситонного резонанса. Угол падения света $30(a)$ и $60^{\circ}(b), s$-поляризация, комнатная температура. 
сти образца), энергия связи экситона $\omega_{b}-\omega_{0}=25$ мэВ, $f_{\text {ex }} / N f_{b}=3$. Погрешность определения $\Gamma_{0}$ мы оцениваем в 0.02 мэВ, а погрешность определения $\Gamma-$ в 5 мэВ. В работе [11] радиационное затухание экситона оценивалось в 0.17 мэВ. Таким образом, несмотря на некоторое различие, одновременная подгонка нескольких оптических спектров подтверждает большое значение радиационного затухания экситона в КЯ InGaN/GaN.

\section{5. Заключение}

В настоящей работе проведено исследование оптических спектров резонансной брэгговской структуры с 60 КЯ InGaN/GaN. В спектрах отражения наблюдается ярко выраженный брэгговский пик, в спектрах пропускания брэгговская особенность более слабая. Совместная подгонка спектров отражения и пропускания, измеренных при двух разных углах падения света, позволила определить параметры экситонов в КЯ более достоверно по сравнению с подгонкой одиночного спектра [11]. Результаты подгонки подтверждают, что радиационное затухание экситонов в КЯ $\mathrm{InGaN} / \mathrm{GaN}$ составляет $\sim 0.2$ мэВ, что значительно больше, чем в других известных системах. Исследование показало также, что для корректного определения параметров экситонов нужно учитывать переходы и между другими уровнями энергетического спектра КЯ.

Работа выполнена при финансовой поддержке РФФИ, грант № 14-29-07243 офи-м.

\section{Список литературы}

[1] Е.Л. Ивченко, А.И. Несвижский, С. Йорда. ФТТ, 36, 2118 (1994).

[2] T. Stroucken, A. Knorr, P. Thomas, S.W. Koch. Phys. Rev. B, 53, 2026 (1996).

[3] V.P. Kochereshko, G.R. Pozina, E.L. Ivchenko, D.R. Yakovlev, A. Waag, W. Ossau, G. Landwehr, R. Hellmann, E.O. Göbel. Superlat. Microstr., 15, 471 (1994).

[4] Y. Merle d'Aubigné, A. Wasiela, H. Mariette, T. Dietl. Phys. Rev. B, 54, 14003 (1996).

[5] G.R. Hayes, J.L. Staehli, U. Oesterle, B. Deveaud, R.T. Phillips, C. Ciuti. Phys. Rev. Lett., 83, 2837 (1999).

[6] M. Hübner, J.P. Prineas, C. Ell, P. Brick, E.S. Lee, G. Khitrova, H.M. Gibbs, S.W. Koch. Phys. Rev. Lett., 83, 2841 (1999).

[7] В.В. Чалдышев, А.С. Школьник, В.П. Евтихиев, Т. Holden. ФТП, 40, 1466 (2006).

[8] D. Goldberg, L.I. Deych, A.A. Lisyansky, Zh. Shi, V.M. Menon, V. Tokranov, M. Yakimov, S. Oktyabrsky. Nat. Photonics, 3, 662 (2009).

[9] V.V. Chaldyshev, Y. Chen, A.N. Poddubny, A.P. Vasil'ev, Z. Liu. Appl. Phys. Lett., 98, 073112 (2011).

[10] В.В. Чалдышев, Е.В. Кунделев, Е.В. Никитина, А.Ю. Егоров, А.А. Горбацевич. ФТП, 46, 1039 (2012).

[11] V.V. Chaldyshev, A.S. Bolshakov, E.E. Zavarin, A.V. Sakharov, W.V. Lundin, A.F. Tsatsulnikov, M.A. Yagovkina, T. Kim, Y. Park. Appl. Phys. Lett., 99, 251103 (2011).
[12] А.С. Большаков, Е.Е. Заварин, А.В. Сахаров, В.В. Лундин, А.Ф. Цацульников, М.А. Яговкина. ФТТ, 55, 1706 (2013).

[13] A.S. Bolshakov, V.V. Chaldyshev, W.V. Lundin, A.V. Sakharov, A.F. Tsatsulnikov, M.A. Yagovkina, E.E. Zavarin. J. Mater. Res., 30, 603 (2015).

[14] Е.Л. Ивченко. ФТТ, 33, 2388 (1991).

[15] P.C. Klipstein, N. Apsley. J. Phys. C: Sol. St. Phys., 19, 6461 (1986).

[16] E.L. Ivchenko. Optical spectroscopy of semiconductor nanostructures (Springer, 2007) p. 97.

Редактор Л.В. Шаронова

\section{Optical spectroscopy of resonant Bragg structure with InGaN/GaN quantum wells}

\author{
A.S. Bolshakov, V.V. Chaldyshev, E.E. Zavarin, \\ A.V. Sakharov, W.V. Lundin, A.F. Tsatsulnikov \\ loffe Institute, \\ 194021 St. Petersburg, Russia
}

\begin{abstract}
Optical reflectance and transmittance spectra of a periodic semiconductor heterostructure with $60 \mathrm{InGaN} / \mathrm{GaN}$ quantum wells were investigated at room temperature. The period of the structure was chosen so that at certain angle of the light incidence it provided a coincidence of the energy of photon resonantly reflected from the Bragg structure with the energy of the quantum well excitons. Parameters of the quantum well excitons were determined by fitting the spectra measured at different angles of incidence, 30 and $60^{\circ}$. We took into account the resonant excitonic transitions in quantum wells as well as the transitions into the continuous spectrum. The radiative decay parameter was determined to be $(0.20 \pm 0.02) \mathrm{meV}$.
\end{abstract}

